\title{
Simian virus 40 may be associated with developing malignant pleural mesothelioma
}

\author{
TRAN DINH THANH ${ }^{1}$, NGUYEN VAN THO $^{2,3}$, NGUYEN SON LAM ${ }^{1}$, \\ NGUYEN HUY DUNG ${ }^{1,2}$, CHIHARU TABATA ${ }^{4}$ and YASUTAKA NAKANO ${ }^{3}$ \\ ${ }^{1}$ Department of Oncology, Pham Ngoc Thach Hospital, Ho Chi Minh City 00848; \\ ${ }^{2}$ Department of Tuberculosis and Lung Diseases, Faculty of Medicine, University of Medicine and Pharmacy at \\ Ho Chi Minh City, Ho Chi Minh City 70000, Vietnam; ${ }^{3}$ Division of Respiratory Medicine, Department of Medicine, \\ Shiga University of Medical Science, Otsu, Shiga 520-2192; ${ }^{4}$ Cancer Center, \\ Hyogo College of Medicine, Nishinomiya, Hyogo 663-8501, Japan
}

Received March 12, 2015; Accepted January 12, 2016

DOI: $10.3892 / \mathrm{ol} .2016 .4174$

\begin{abstract}
Malignant pleural mesothelioma (MPM) is associated with a history of heavy, long-term exposure to asbestos. However, MPM may also be associated with simian virus 40 (SV40), a polyomavirus. The association between SV40 and MPM remains unclear. The present study was conducted in order to investigate the proportion of SV40 presence in the histological specimens of Vietnamese patients with MPM. Histological specimens were obtained from 45 patients (19 men and 26 women) with MPM at the Pham Ngoc Thach Hospital in Ho Chi Minh City, Vietnam. The specimens were processed and examined in order to detect the presence of the SV40 large T antigen (SV40 Tag) expression using immunohistochemistry. Of the 45 patients, 23 (51\%) were epithelioid, 7 (16\%) were biphasic, 6 (13\%) were sarcomatoid, 4 (9\%) were desmoplastic, 4 (9\%) were well-differentiated papillary and $1(2 \%)$ was the anaplastic subtype. In total, $9 / 45$ patients $(20 \%)$ demonstrated SV40 Tag expression. The proportion of patients that demonstrated SV40 Tag expression was not significantly different between the epithelioid subtype and the other subtypes ( 22 vs. $18 \%$; $\mathrm{P}=1.000$ ) or between the patients with stage IV disease and other stages ( 20 vs. $20 \%$; $\mathrm{P}=1.000)$. The median survival time was not significantly different between the patients with or without SV40 Tag expression (196 vs. 236 days, $\mathrm{P}=0.8949$ ). In summary, a 5 th of the Vietnamese patients with MPM were associated with infection with SV40. SV40 may be a potential cause of MPM in Vietnam and this potential association requires additional studies.
\end{abstract}

Correspondence to: Dr Nguyen Van Tho, Department of Tuberculosis and Lung Diseases, Faculty of Medicine, University of Medicine and Pharmacy at Ho Chi Minh City, 217 Hong Bang Street, Ho Chi Minh City 70000, Vietnam

E-mail: thonguyen0225@gmail.com

Key words: asbestos, pleural, immunohistochemistry, malignant mesothelioma, simian virus 40

\section{Introduction}

Malignant mesothelioma is a rare but fatal disease that arises from the epithelial lining of the pleura, peritoneum, pericardium and tunica vaginalis. Malignant pleural mesothelioma (MPM) is the most common form, accounting for $80-90 \%$ of malignant mesotheliomas $(1,2)$. A history of heavy and long-term exposure to asbestos is the established cause of MPM (3). However, MPM may result from other factors, including genetics, erionite, radiation and simian virus 40 (SV40), which may work alone or in combination (4). SV40 is a polyomavirus of which the natural hosts are rhesus monkeys. SV40 may infect human mesothelial cells, and may transform the cells using a mechanism whereby the tumor antigens, large $\mathrm{T}$ antigen (Tag) and small $\mathrm{t}$ antigen (tag), bind and inactivate the cellular tumor suppressors tumor protein p53 and retinoblastoma 1 . These interactions may contribute to the development of malignant mesotheliomas by rendering mesothelial cells more susceptible to other carcinogens (5-7).

The role of SV40 in the pathogenesis of MPM remains unclear $(1,2,4)$. Certain studies have detected SV40 DNA sequences or SV40 Tag in mesothelioma cells (8-10), but others have not (11-14). Geographical variation may be one reason for the discrepancy in SV40 detection, as SV40-contaminated polio vaccines, which had varied availability between countries, have been suspected as a major source of human infection (15). The association between SV40 and MPM remains unclear. The interaction between SV40 and asbestos exposure in the pathogenesis of MPM is unknown. The present study was conducted in order to investigate the proportion of SV40 presence in the histological specimens of Vietnamese patients with MPM.

\section{Materials and methods}

The present retrospective study was conducted at Pham Ngoc Thach Hospital, a referral chest hospital, in Ho Chi Minh City, Vietnam. The study protocol was approved by the Ethics Committee of the hospital.

Patients. The records of patients that were diagnosed with MPM between January 2008 and June 2012 were searched 
for on the patient database of the Department of Pathology, Pham Ngoc Thach Hospital. The medical records and histological specimens of the patients were archived. The patients or close relatives of the patients were asked to participate in the study, and all participants provided informed written consent. Patients that met the following criteria were enrolled: i) Definitively diagnosed as MPM; ii) the formaldehyde-fixed, paraffin-embedded tissues of the pleural specimens were eligible for additional immunohistochemical analysis; and iii) the patients or close relatives were available for a face-to-face or telephone interview. Patients were excluded for the following reasons: i) The formaldehyde-fixed, paraffin-embedded tissues of the pleural specimens were not eligible for immunohistochemical analysis due to small size or a lack of tumor tissue; and ii) the patients or the close relatives were not available or contactable.

Patients were definitively diagnosed as MPM based on histological examinations and immunohistochemical staining $(16,17)$. In total, 4 positive markers, including calretinin, desmin, monoclonal mouse anti-human mesothelial cell clone HBME-1 and Wilms tumor 1 were used to definitively diagnose MPM. Various negative markers were used to rule out other cancers metastasized to the pleura, including: Keratin 7, carcinoembryonic antigen, transcription termination factor, RNA polymerase I and epidermal growth factor receptor for adenocarcinoma; enolase 2, gamma neuronal, synaptrophysin and mouse monoclonal EpCAM antibody for small cell lung cancer; and clathrin, light chain A, cluster of differentiation (CD)3, CD20, CD30, CD68 and myeloperoxidase for lymphoma and leukemia.

Patients or close relatives were interviewed in order to determine a history of asbestos exposure. A history of asbestos exposure was designated to patients that had ever lived in a fiber cement-roofed house or worked in asbestos-associated industries, including the manufacture of fiber cement, ceramic tiles, insulating materials or other construction materials, shipbuilding and mineral mining.

All patients were followed up until August 31, 2013 to determine the survival time. The survival time was measured between the date of clinical diagnosis and mortality or censoring (the last date the patients were lost to follow-up or the last date the patients could be contacted, whether they remained alive or not). The date of clinical diagnosis was defined as the date on which MPM was diagnosed at Pham Ngoc Thach Hospital.

Detection of SV4O Tag expression. The formaldehyde-fixed, paraffin-embedded tissues of the pleural specimens of the patients were immunostained for SV40 Tag expression. The Lab Vision mouse monoclonal antibody pAb101 (dilution, 1:100; catalog no., MS-1832-P; Thermo Fisher Scientific Inc., Waltham, MA, USA) was used to detect SV40 Tag expression. The secondary antibody used was Lab Vision ${ }^{\mathrm{TM}}$ biotinylated goat anti-polyvalent anti-mouse/rabbit immunoglobulin $\mathrm{G}$ (ready to use; catalog no., TP-125-BN; Thermo Fisher Scientic, Inc.). The detection system used was a Thermo Scientific ${ }^{\mathrm{TM}}$ Lab Vision $^{\mathrm{TM}}$ DAB Plus Substrate System (Thermo Fisher Scientific, Inc.). The staining procedure was performed according to the manufacturer's protocol. Specimens with nuclear immunoreactive tumor cells were considered to express SV40 Tag (Fig. 1). The positive results were scored according to the Allred score system as follows: $1-25 \%$ of tumor cells demonstrate SV40 Tag expression, 1+; 26-50\%, $2^{+}$; and $>50 \%, 3^{+}(18)$.

Detection of asbestos bodies. A light microscope (ECLIPSE 50i; Nikon Corp., Tokyo, Japan) was used to examine and count the presence of asbestos bodies in specimens of lung tissue or bronchoalveolar lavage fluid. The lung tissue specimens were stained using hematoxylin-eosin (Thermo Fisher Scientific, Inc.). The fluid specimens were stained using the papanicolaou method (19) (Fig. 2), subsequent to breaking down the mucus in the fluid using $10 \% \mathrm{NaOH}$ (Thermo Fisher Scientific, Inc.). A specimen with $>5$ asbestos bodies in every 10 examined fields with a $\mathrm{x} 40$ magnification was considered to contain asbestos bodies (20).

Statistical analysis. The categorical variables are expressed as frequency and percent. The comparisons of the proportions of SV40 Tag expression between 2 groups were examined using the Fisher's exact test. Comparisons of the survival time between two groups were examined using the Log-Rank test of the Kaplan-Meier analysis. The Cox regression survival analysis was used to examine the effect of chemotherapy on the survival time, adjusting for the clinical stages of MPM. A P-value of $<0.05$ was considered to indicate a statistically significant difference. All statistical analyses were performed using the JMP 9.0.2 statistical software (SAS Institute Inc., Cary, NC, USA).

\section{Results}

Study population. Of the patients diagnosed with MPM at Pham Ngoc Thach Hospital between January 2008 and June 2012, 45 patients met the inclusion criteria. The mean $( \pm$ standard deviation) and median age were $59( \pm 15)$ and 58 years, respectively. The youngest patient was 25 and the eldest was 88 years old. Over half of the patients were female and $89 \%$ were in stage IV disease (Table I). Only $44 \%$ of the patients had a history of asbestos exposure. For the definitive diagnosis of MPM, $60 \%$ of the patients required a transcutaneous needle biopsy, while $40 \%$ required a thoracoscopic biopsy. Epithelioid was the most common histological subtype (51\%) of MPM. Only 22 patients had clinical specimens available for the examination of asbestos bodies, 21 of which possessed bronchoalveolar lavage fluid specimens and 1 of which possessed a lung tissue specimen. Asbestos bodies were identified in $10(45 \%)$ out of 22 patients.

Proportion of SV40 Tag expression. In total, 9 (20\%) out of 45 patients exhibited SV40 Tag expression in the histological specimens: 2 patients with an Allred score of $1^{+}, 4$ patients with an Allred score of $2^{+}$; and 3 patients with an Allred score of $3^{+}$. However, only $1(5 \%)$ out of 22 patients exhibited SV40 Tag expression and asbestos bodies.

The proportion of SV40 Tag expression was decreased in males compared with females (5 vs. $31 \%$ ), but this difference was not significant $(\mathrm{P}=0.0578)$. Similarly, the proportion of SV40 Tag expression was not significantly different between the patients with and without asbestos bodies $(\mathrm{P}=0.5940)$, 
Table I. Characteristics of 45 patients with malignant pleural mesothelioma.

\begin{tabular}{lcc}
\hline Characteristic & No. of patients & \% of total \\
\hline Gender & 19 & 42 \\
Male & 26 & 58 \\
Female & & \\
History of asbestos exposure & 20 & 44 \\
Yes & 25 & 56 \\
No & & \\
Method of pleural biopsy & 27 & 60 \\
Transcutaneous needle & 18 & 40 \\
Thoracoscopy & & \\
Histological subtypes & 23 & 51 \\
Epithelioid & 7 & 16 \\
Biphasic & 6 & 13 \\
Sarcomatoid & 4 & 9 \\
Desmoplastic & 4 & 9 \\
Papillary & 1 & 2 \\
Anaplastic & & \\
SV40 Tag expression & 9 & 20 \\
Positive & 36 & 80 \\
Negative & & \\
Asbestos bodies & 10 & 45 \\
Positive & 12 & 55 \\
Negative & & \\
Clinical stage & 3 & 4 \\
II & 2 & \\
III & 40 & \\
IV & 40 & \\
\hline SV & & \\
\hline
\end{tabular}

SV40 Tag, large T antigen of simian virus 40 .

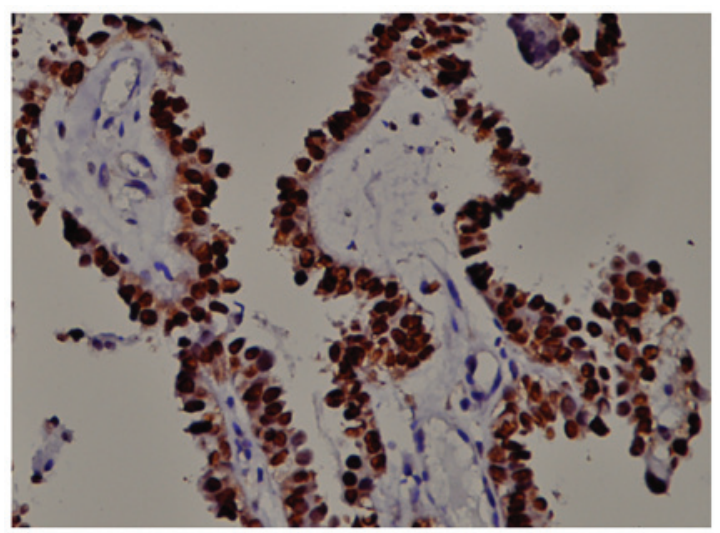

Figure 1. Expression of simian virus 40 large $\mathrm{T}$ antigen was assessed by immunohistochemical staining using the pAb101 antibody. The nuclei of the tumor cells are strongly immunoreactive (brown color; original magnification, $\mathrm{x} 100)$. The specimen in the present figure was provided an Allred score of $3^{+}$.

with the epithelioid subtype and other subtypes $(\mathrm{P}=1.000)$, or the patients with stage IV and other stages of disease $(\mathrm{P}=1.000)$ (Table II). There was no significant difference in

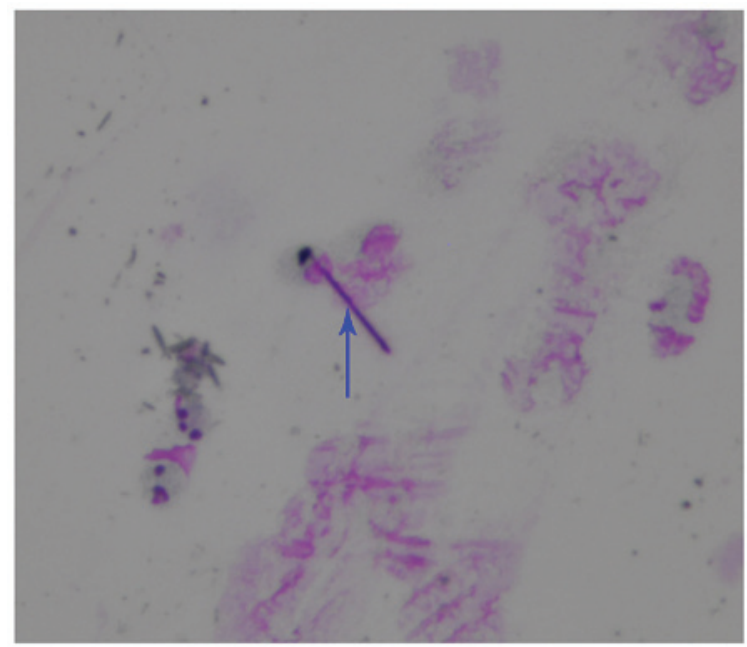

Figure 2. The presence of an asbestos body (blue arrow) in a specimen of bronchoalveolar lavage fluid (papanicolaou stain; original magnification, $\mathrm{x} 100)$.

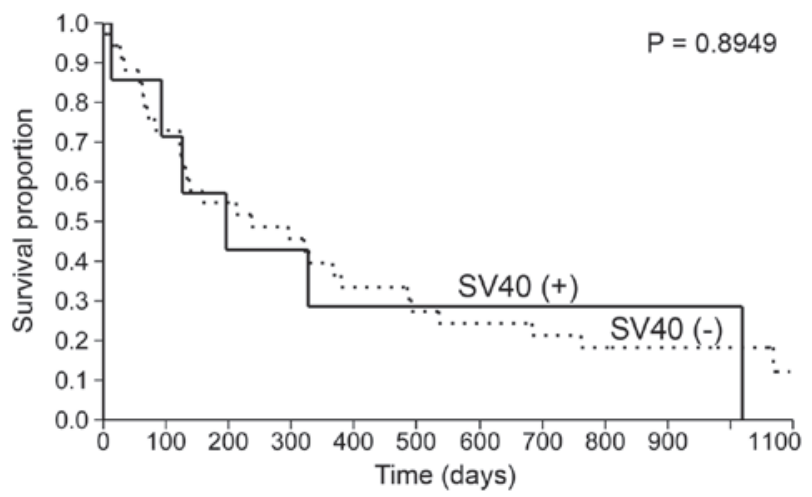

Figure 3. The Kaplan-Meier survival curves of the patients with (solid line) and without (dotted line) SV40 Tag expression. The median survival time was not significantly different between the 2 groups.

the mean age between the patients with and without SV40 Tag expression (58.8 \pm 16.6 vs. $59.1 \pm 14.6 ; \mathrm{P}=0.7227)$.

Survival time. Among the 45 patients, 34 succumbed to the disease, 5 dropped out of the study and 6 survived during the follow-up. The median survival time was 236 days [95\% confidence interval (CI), 125-366]. The proportions of patients surviving for 1 and 2 years were 35\% (95\% CI, 22-51\%) and 23\% (95\% CI, 12-38\%), respectively. The median survival time was not significantly different between the patients with or without SV40 Tag expression (196 vs. 236 days; $\mathrm{P}=0.8949$ ) (Table III; Fig. 3). Similarly, the median survival time was not significantly different between the patients with the epithelioid subtype and other subtypes ( 327 vs. 131 days; $P=0.7803$ ). By contrast, the median survival time was significantly increased in the patients receiving chemotherapy compared with the patients not receiving chemotherapy (435 vs. 196 days; $\mathrm{P}=0.0397$ ) (Fig. 4A). The mortality in the patients receiving chemotherapy decreased by $52 \%$ compared with the patients not receiving chemotherapy (hazard ratio, 0.48; 95\% CI, 0.23-0.96) (Table III). However, the Cox regression survival analysis indicated that only the clinical stages of MPM had significant effect on the survival 
Table II. Comparison of the proportions of SV40 Tag expression between groups.

SV40 Tag expression

Characteristic

Expressed, frequency (\%)

Not expressed, frequency (\%)

P-value

Gender

Male

$1 / 19(5)$

$18 / 19(95)$

0.0578

Female

$8 / 26(31)$

$18 / 26(69)$

Asbestos bodies

Positive

$1 / 10(10)$

$9 / 10(90)$

0.5940

Negative

$3 / 12(25)$

9/12 (75)

Histological subtype

Epithelioid $\quad 5 / 23(22)$

$18 / 23(78)$

1.0000

Other

$4 / 22(18)$

$18 / 22(82)$

Stage

Stage IV

$8 / 40(20)$

$32 / 40(80)$

Stages II-III

$1 / 5(20)$

$4 / 5(80)$

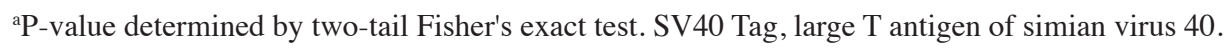

Table III. Comparisons of the survival time between groups. The total number of patients was 45 .

\begin{tabular}{|c|c|c|c|c|c|}
\hline Characteristic & $\begin{array}{l}\text { No. of } \\
\text { cases }\end{array}$ & $\begin{array}{c}\text { No. of } \\
\text { mortalities }\end{array}$ & $\begin{array}{c}\text { No. of } \\
\text { survivors, } \mathrm{n}(\%)\end{array}$ & $\begin{array}{l}\text { Median survival time, } \\
\text { days }(95 \% \mathrm{CI})\end{array}$ & P-value \\
\hline SV40 Tag expression & & & & & 0.8949 \\
\hline Expressed & 9 & 6 & $3(33)$ & $196(13-1,019)$ & \\
\hline Not expressed & 36 & 28 & $8(22)$ & $236(122-379)$ & \\
\hline Histological subtype & & & & & 0.7803 \\
\hline Epithelioid & 23 & 19 & $4(17)$ & $327(125-491)$ & \\
\hline Other & 22 & 15 & $7(32)$ & $131(62-485)$ & \\
\hline Management & & & & & $0.0397^{\mathrm{b}}$ \\
\hline Chemotherapy & 17 & 12 & $5(29)$ & $435(125-1,019)$ & \\
\hline No chemotherapy & 28 & 22 & $6(21)$ & $196(62-327)$ & \\
\hline
\end{tabular}

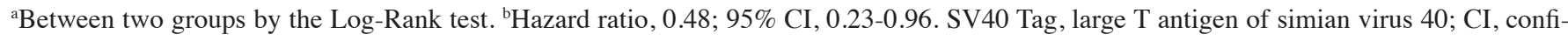
dence interval.

A

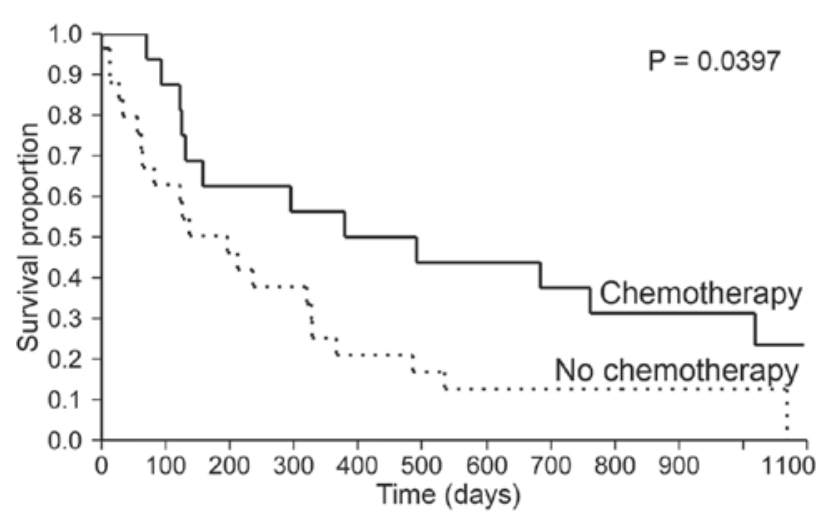

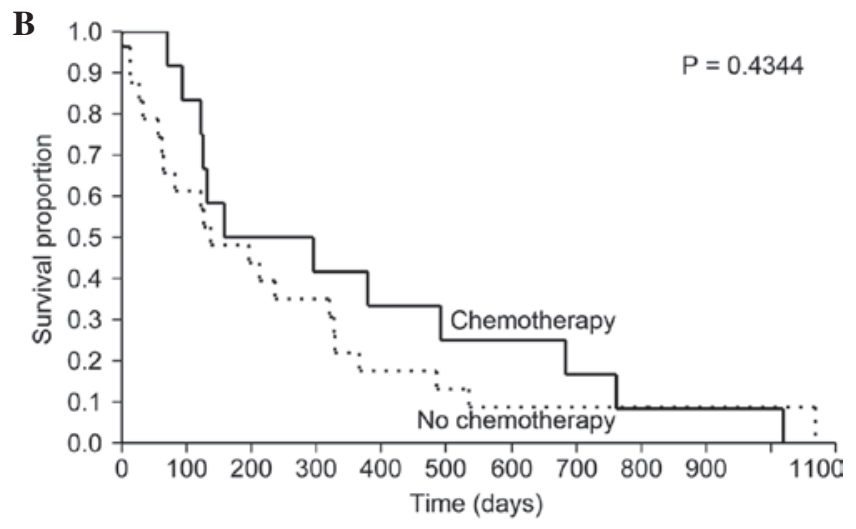

Figure 4. The Kaplan-Meier survival curves of the patients receiving chemotherapy (solid line) and the patients not receiving chemotherapy (dotted line). (A) All patients. Median survival time was significantly increased in the patients receiving chemotherapy compared with patients not receiving chemotherapy. (B) A subset of patients with stage IV disease. Median survival time was not significantly different between the patients receiving chemotherapy and the patients not receiving chemotherapy. 
time $(\mathrm{P}<0.0001)$, while the chemotherapy did not (adjusted hazard ratio, $0.75 ; 95 \% \mathrm{CI}, 0.36-1.52 ; \mathrm{P}=0.4317$ ). In a subset of patients with stage IV disease, the median survival time was not significantly different between the patients receiving chemotherapy and the patients not receiving chemotherapy (227 vs. 137 days; $\mathrm{P}=0.4344$ ) (Fig. 4B).

\section{Discussion}

The present study shows that a 5 th of the Vietnamese patients with MPM demonstrated SV40 Tag expression in their histological specimens. This finding indicates that a 5 th of patients with MPM may be associated with SV40, which may explain why not all patients with MPM are associated with asbestos exposure. Only half of the patients had evident asbestos exposure, indicated by either the history of asbestos exposure or the examination of asbestos bodies in clinical specimens.

To the best of our knowledge, the present study is the first to propose the association between SV40 and MPM in Vietnamese patients and to register Vietnam as one of the countries in which SV40 may potentially affect the pathogenesis of MPM. However, the proportion of patients with SV40 in the present study is decreased compared with previously published studies $(8,10,15)$. This finding may result from the varying prevalence of SV40 infection between countries. The immunohistochemical methods used in the present study may also not be as sensitive as the molecular methods of SV40 detection, used in previous studies $(9,11)$.

For the patients that demonstrated SV40 Tag expression, the means by which they contracted the virus was unknown. In previous studies, SV40 infection was hypothesized to be a result of receiving the SV40-contaminated polio vaccines that were produced before $1961(8,10,15)$. In the present study, only 2 patients with SV40 Tag expression were born prior to 1961. Therefore, the remaining patients may have possibly contracted the virus through SV40-contaminated polio vaccines that remained available in Vietnam subsequent to 1961, as was observed in other Eastern European countries (21). Another explanation is that the patients were infected by other unknown sources (22).

In the present study, only half of the patients exhibited evidence of asbestos exposure, which is a lower figure compared with other reports (70-80\%) (3). There are several potential reasons for this low prevalence: The method used to detect asbestos bodies may not be sensitive enough (23); only $22(49 \%)$ out of 45 patients had clinical specimens available for asbestos body examination; and there may be a recalled bias regarding the history of asbestos exposure during the interviews of patients or close relatives.

Notably, only $5 \%$ of the patients exhibited overlapping results for the presence of asbestos bodies and SV40 Tag expression. This finding may imply that the interaction between SV40 and asbestos exposure is not the prerequisite for the development of mesothelioma in humans, which has been previously demonstrated in hamsters (24). The finding also supports the speculation that SV40 may be an independent carcinogen (25) or a co-carcinogen, and interact with other environmental or genetic factors in the pathogenesis of malignant mesothelioma (6).
The proportion of patients that survived for 1 and 2 years in the present study was similar to other populations $(26,27)$. The finding that only the clinical stages of MPM significantly affected the survival time, whereas chemotherapy did not, partly explains why the prognosis of MPM remains poor, regardless of current therapies for MPM, particularly as the majority of MPM patients are diagnosed at stage IV of disease. In the present study, the median survival time was not significantly different between the patients with or without SV40 Tag expression. This finding may be explained by the lack of significant differences in the mean age, histological subtypes and clinical stages of MPM between the 2 groups of patients (Table II).

There are certain strengths of the present study. First, the present study is the first to propose the association between SV40 and MPM in Vietnam. Second, the present study included a balanced number of male and female patients, making the results more generalizable compared with other studies. Third, SV40 detection was based on immunohistochemical analysis, which avoids the potential false expression that may occur in polymerase chain reaction tests due to the presence of SV40 sequence-contaminated plasmids in pathological laboratories (13).

However, the present study has certain limitations. First, as the present study is retrospective, not all patients had clinical specimens available for asbestos body examination. Therefore, the prevalence of asbestos exposure may be underestimated in the present study. Second, SV40 was only detected using immunohistochemistry, which may not be as sensitive as other molecular methods. Third, immunohistochemistry results may yield false expression due to the immunostaining procedure and result interpretation. However, the immunostaining procedure was performed following the antibody manufacturer's protocols to minimise the risk of false expression. In addition, SV40 expression was strictly defined as the presence of strong immunoreactive tumor nuclei, which indicates the nuclear expression of SV40 Tag. Therefore, the possibility of false expression may be limited. Fourth, since the sample size is relatively small, the power to detect statistically significant differences between groups of patients was not sufficient. Finally, the present study is limited by the lack of published data regarding the general levels of SV40 in the Vietnamese population.

In conclusion, a 5 th of the Vietnamese patients with MPM were infected with SV40. SV40 may be another potential cause of MPM in Vietnam and this potential association requires thorough investigation with a larger sample size and more reliable methods of SV40 detection.

\section{References}

1. Robinson BM: Malignant pleural mesothelioma: An epidemiological perspective. Ann Cardiothorac Surg 1: 491-496, 2012.

2. Robinson BW, Musk AW and Lake RA: Malignant mesothelioma. Lancet 366: 397-408, 2005.

3. Cugell DW and Kamp DW: Asbestos and the pleura: A review. Chest 125: 1103-1117, 2004.

4. Yang H, Testa JR and Carbone M: Mesothelioma epidemiology, carcinogenesis, and pathogenesis. Curr Treat Options Oncol 9: 147-157, 2008.

5. Carbone M, Pannuti A, Zhang L, Testa JR and Bocchetta M: A novel mechanism of late gene silencing drives SV40 transformation of human mesothelial cells. Cancer Res 68: 9488-9496, 2008. 
6. Carbone M, Pass HI, Miele L and Bocchetta M: Novel developments about the association of SV40 with human mesothelioma. Oncogene 22: 5173-5180, 2003.

7. Jaurand MC and Fleury-Feith J: Pathogenesis of malignant pleural mesothelioma. Respirology 10: 2-8, 2005.

8. Comar M, Rizzardi C, de Zotti R, Melato M, Bovenzi M, Butel JS and Campello C: SV40 multiple tissue infection and asbestos exposure in a hyperendemic area for malignant mesothelioma. Cancer Res67: 8456-8459, 2007.

9. Jin M, Sawa H, Suzuki T, Shimizu K, Makino Y, Tanaka S, Nojima T, Fujioka Y, Asamoto M, Suko N, et al: Investigation of simian virus 40 large $\mathrm{T}$ antigen in 18 autopsied malignant mesothelioma patients in Japan. J Med Virol 74: 668-676, 2004.

10. Testa JR, Carbone M, Hirvonen A, Khalili K, Krynska B Linnainmaa K, Pooley FD, Rizzo P, Rusch V and Xiao GH: A multi-institutional study confirms the presence and expression of simian virus 40 in human malignant mesotheliomas. Cancer Res 58: 4505-4509, 1998

11. Aoe K, Hiraki A, Murakami T, Toyooka S, Shivapurkar N, Gazdar AF, Sueoka N, Taguchi K, Kamei T, Takeyama H, et al: Infrequent existence of simian virus 40 large $\mathrm{T}$ antigen DNA in malignant mesothelioma in Japan. Cancer Sci 97: 292-295, 2006.

12. Hirvonen A, Mattson K, Karjalainen A, Ollikainen $\mathrm{T}$, Tammilehto L, Hovi T, Vainio H, Pass HI, Di Resta I, Carbone M and Linnainmaa K: Simian virus 40 (SV40)-like DNA sequences not detectable in finnish mesothelioma patients not exposed to SV40-contaminated polio vaccines. Mol Carcinog 26: 93-99, 1999.

13. López-Ríos F, Illei PB, Rusch V and Ladanyi M: Evidence against a role for SV40 infection in human mesotheliomas and high risk of false-positive PCR results owing to presence of SV40 sequences in common laboratory plasmids. Lancet 364 1157-1166, 2004.

14. Pilatte Y, Vivo C, Renier A, Kheuang L, Greffard A and Jaurand MC: Absence of SV40 large T-antigen expression in human mesothelioma cell lines. Am J Respir Cell Mol Biol 23 788-793, 2000

15. De Rienzo A, Tor M, Sterman DH, Aksoy F, Albelda SM and Testa JR: Detection of SV40 DNA sequences in malignant mesothelioma specimens from the United States, but not from Turkey. J Cell Biochem 84: 455-459, 2002.
16. Kushitani K, Takeshima Y, Amatya VJ, Furonaka O, Sakatani A and Inai K: Immunohistochemical marker panels for distinguishing between epithelioid mesothelioma and lung adenocarcinoma. Pathol Int 57: 190-199, 2007.

17. Sandeck HP, Røe OD, Kjærheim K, Willén H and Larsson E: Re-evaluation of histological diagnoses of malignant mesothelioma by immunohistochemistry. Diagn Patho 5: 47, 2010

18. Allred DC, Harvey JM, Berardo M and Clark GM: Prognostic and predictive factors in breast cancer by immunohistochemical analysis. Mod Pathol 11: 155-168, 1998.

19. Nguyen GK and Kline TS (eds): Cytology laboratory and quality assurance practice. In: Essentials of Exfoliative Cytology. Igaku-Shoin Medical Publishers, Inc., New York, NY, pp6-13, 1992.

20. De Vuyst P, Dumortier P, Moulin E, Yourassowsky N, Roomans P, de Francquen P and Yernault JC: Asbestos bodies in bronchoalveolar lavage reflect lung asbestos body concentration. Eur Respir J 1: 362-367, 1988.

21. Cutrone R, Lednicky J, Dunn G, Rizzo P, Bocchetta M, Chumakov K, Minor P and Carbone M: Some oral poliovirus vaccines were contaminated with infectious SV40 after 1961. Cancer Res 65: 10273-10279, 2005.

22. Martini F, Corallini A, Balatti V, Sabbioni S, Pancaldi C and Tognon M: Simian virus 40 in humans. Infect Agent Cancer 2: $13,2007$.

23. Xaubet A, Rodriguez-Roisín R, Bombí JA, Marín A, Roca J and Agustí-Vidal A: Correlation of bronchoalveolar lavage and clinical and functional findings in asbestosis. Am Rev Respir Dis 133: 848-854, 1986

24. Kroczynska B, Cutrone R, Bocchetta M, Yang H, Elmishad AG, Vacek P, Ramos-Nino M, Mossman BT, Pass HI and Carbone M: Crocidolite asbestos and SV40 are cocarcinogens in human mesothelial cells and in causing mesothelioma in hamsters. Proc Natl Acad Sci USA 103: 14128-14133, 2006.

25. Cicala C, Pompetti F and Carbone M: SV40 induces mesotheliomas in hamsters. Am J Pathol 142: 1524-1533, 1993.

26. Milano MT and Zhang H: Malignant pleural mesothelioma: A population-based study of survival. J Thorac Oncol 5: 1841-1848, 2010.

27. van der Bij S, Koffijberg H, Burgers JA, Baas P, van de Vijver MJ, de Mol BA and Moons KG: Prognosis and prognostic factors of patients with mesothelioma: A population-based study. Br J Cancer 107: 161-164, 2012. 